\title{
Blood Eosinophil Count and Metabolic, Cardiac and Pulmonary Outcomes: A Mendelian Randomization Study
}

\author{
Marzyeh Amini, ${ }^{1}$ Judith M. Vonk, ${ }^{1,2}$ Ali Abbasi, ${ }^{1}$ Bram P. Prins, ${ }^{1}$ Marcel Bruinenberg, ${ }^{3}$ Lude Franke, ${ }^{4}$ \\ Pim van der Harst, ${ }^{5}$ Gerjan Navis, ${ }^{6}$ Gerard H. Koppelman, ${ }^{2,7}$ Bruce H. R. Wolffenbuttel, ${ }^{8}$ \\ H. Marike Boezen, ${ }^{1,2}$ Harold Snieder, ${ }^{1}$ Daniel I. Chasman, ${ }^{9}$ and Behrooz Z. Alizadeh ${ }^{1}$ \\ ${ }^{1}$ Department of Epidemiology, University Medical Center Groningen, University of Groningen, Groningen, The \\ Netherlands \\ ${ }^{2}$ Groningen Research Institute for Asthma and COPD (GRIAC), University Medical Center Groningen, University of \\ Groningen, Groningen, The Netherlands \\ ${ }^{3}$ LifeLines Cohort Study, University Medical Center Groningen, University of Groningen, Groningen, The Netherlands \\ ${ }^{4}$ Department of Genetics, University Medical Center Groningen, University of Groningen, Groningen, The Netherlands \\ ${ }^{5}$ Department of Cardiology, University Medical Center Groningen, University of Groningen, Groningen, The Netherlands \\ ${ }^{6}$ Department of Nephrology, University Medical Center Groningen, University of Groningen, Groningen, The Netherlands \\ ${ }^{7}$ Department of Pediatric Pulmonology and Pediatric Allergology, Beatrix Children's Hospital, University Medical Center \\ Groningen, University of Groningen, Groningen, The Netherlands \\ ${ }^{8}$ Department of Endocrinology, University Medical Center Groningen, University of Groningen, Groningen, The \\ Netherlands \\ ${ }^{9}$ Division of Preventive Medicine, Brigham and Women's Hospital, Boston, MA, USA
}

\begin{abstract}
Blood eosinophil count is associated with a variety of common complex outcomes in epidemiological observation. The aim of this study was to explore the causal association between determined blood eosinophil count and 20 common complex outcomes ( 10 metabolic, 6 cardiac, and 4 pulmonary). Through Mendelian randomization, we investigated genetic evidence for the genetically determined eosinophil in association with each outcomes using individual-level LifeLines cohort data $(n=13,301)$, where a weighted eosinophil genetic risk score comprising five eosinophil associated variants was created. We further examined the associations of the genetically determined eosinophil with those outcomes using summary statistics obtained from genome-wide association study consortia (6 consortia and 14 outcomes). Blood eosinophil count, by a 1-SD genetically increased, was not statistically associated with common complex outcomes in the LifeLines. Using the summary statistics, we showed that a higher genetically determined eosinophil count had a significant association with lower odds of obesity (odds ratio (OR) $0.81,95 \%$ confidence interval (CI) $[0.74$, 0.89]) but not with the other traits and diseases. To conclude, an elevated eosinophil count is unlikely to be causally associated to higher risk of metabolic, cardiac, and pulmonary outcomes. Further studies with a stronger genetic risk score for eosinophil count may support these results.
\end{abstract}

- Keywords: eosinophil count, genetic risk score, Mendelian randomization, instrumental variable, complex diseases, metabolic diseases, cardiovascular diseases, pulmonary diseases

Emerging epidemiological studies have indicated that a higher count of eosinophils, as specialized multifunctional leukocytes, in the blood is associated with a higher risk of complex diseases in the human population (Amini et al., 2016; Babio et al., 2013; Bafadhel et al., 2012; Kim, Noh et al., 2008; Kim, Choi et al., 2006; Meng et al., 2012; Shim et al., 2006; Siva et al., 2007; Wu et al., 2011). In the population-based LifeLines study in the north of the Netherlands, we have recently shown that an increased blood eosinophil count correlates with a higher risk of metabolic and pulmonary diseases (Amini et al., 2016).
The association of eosinophil count with common complex outcomes may be due to the presence of confounding

RECEIVED 26 January 2018; ACCEPTED 29 January 2018. First published online 6 March 2018.

ADDRess For CORRESPONDENCE: Marzyeh Amini MSc PhD Fellow, Department of Epidemiology, University Medical Center Groningen, University of Groningen, Hanzeplein 1, PO Box 30001, 9700 RB Groningen, The Netherlands.

E-mail:m.amini@umcg.nl 
factors or reverse causation, or also may be consistent with a causal role of eosinophil in these traits and diseases (Astle et al., 2016; Davey Smith \& Ebrahim, 2005; Davey Smith \& Hemani, 2014). If a causal association exists, then common genetic variants associated with eosinophil count may influence these complex outcomes. A previous genomewide association study (GWAS) has identified five genetic variants associated with eosinophil count in European and Asian populations (Gudbjartsson et al., 2009). Interestingly, among the five genetic variants, the rs3184504* $\mathrm{T}$ variant (mapped to $\mathrm{SH} 2 \mathrm{~B} 3$ gene) allele has been shown to be associated with a higher risk of myocardial infarction (MI), and the rs $1420101^{\star} \mathrm{A}$ variant (mapped to IL1RL1 gene) with a higher risk of asthma (Gudbjartsson et al., 2009). Other studies also indicated the associations of some eosinophil genetic variants with complex outcomes (Aghabozorg Afjeh et al., 2014; Lian et al., 2013; Savenije et al., 2011; Talmud et al., 2009) and the causal role of eosinophil in autoimmune diseases (Astle et al., 2016). However, the causality of associations between eosinophil count and other common complex diseases and their related intermediate traits remained unclear. An intermediate trait is a measurable component that lies along the pathway of diseases, which is not a risk factor rather it manifests the underlying disease.

Implementation of Mendelian randomization (MR) methodology (Davey Smith \& Hemani, 2014; Didelez \& Sheehan, 2007) may clarify the potential causal association between eosinophil count and risk of complex outcomes by transition from healthy intermediate traits to related diseases. Using the genetic risk score of five constituting eosinophil genetic variants (eosinophil genetic risk score (eos-GRS)), the explanatory effects of genetically determined eosinophil on the risk of complex diseases can be investigated. Recently, we successfully applied the MR methodology to characterize the causal association between C-reactive protein (CRP) and complex outcomes (Prins et al., 2016), as well as between bilirubin and type 2 diabetes (T2D) (Abbasi et al., 2015). In this study, using a similar methodology, we performed MR, using two approaches to assess how a genetically determined eosinophil count contributes causally to the risk of 20 complex intermediate traits and diseases in three classes of metabolic, cardiac, and pulmonary outcomes. In the first approach, we performed MR analysis using individual-level data. In the second approach, in order to replicate the findings of the first approach in an expanded sample size, we applied MR analysis to the summary statistics acquired from six large-scale GWAS consortia of eosinophil and 14 out of 20 complex traits and diseases (Figure 1).

\section{Material and Methods}

\section{Study Design}

To estimate the causal association of eosinophil count on outcomes of interest, we performed MR analysis using weighted eos-GRSs. First, we applied MR analysis procedures to the individual-level LifeLines cohort data, adjusted for age and gender, to assess causal inference on 20 complex intermediate traits and diseases. Second, we tested the causal association between eos-GRS and 14 outcomes of interest from 6 consortia data (Figure 1).

\section{Outcomes}

We focused on three classes of outcomes, including metabolic, cardiac, and pulmonary outcomes. The first class of metabolic outcomes comprised body mass index (BMI, $\mathrm{kg} / \mathrm{m}^{2}$ ), triglycerides (TG, mmol/L), total cholesterol (TC, mmol/L), high-density lipoprotein cholesterol (HDL$\mathrm{C}, \mathrm{mmol} / \mathrm{L}$ ), low-density lipoprotein cholesterol (LDL-C, $\mathrm{mmol} / \mathrm{L}$ ), hemoglobin A1c (HbA1c, \%), fasting glucose (FG, mmol/L), obesity, metabolic syndrome (MetS), and T2D. The second class of cardiac outcomes included systolic blood pressure (SBP, $\mathrm{mmHg}$ ), diastolic blood pressure (DBP, $\mathrm{mmHg}$ ), mean arterial pressure (MAP, mmHg), pulse pressure (PP, mmHg), hypertension (HTN), and MI. The third class of pulmonary outcomes included forced expiratory volume in one second $\left(\mathrm{FEV}_{1}, \mathrm{~L}\right)$, ratio of $\mathrm{FEV}_{1}$ and forced vital capacity $\left(\mathrm{FEV}_{1} / \mathrm{FVC}\right)$, chronic obstructive pulmonary disease (COPD), and asthma.

\section{Genetic Variants and GRS}

Five genetic variants affecting eosinophil count were selected, based on a GWAS discovery of over 21,000 European individuals (Supplementary Table S1; Gudbjartsson et al., 2009). The weighted GRS was estimated for each individual in LifeLines by summing the product of multiplying the number of risk alleles at each locus (range 0-2) with the corresponding effect sizes obtained from the largest meta GWAS analysis on eosinophil count, as given in Table S1 (Gudbjartsson et al., 2009).

\section{Data Analyses}

In order to assess the causal association of eosinophil on complex traits and diseases, we performed MR analyses using eos-GRSs as instrumental variables (Abbasi et al., 2015; Prins et al., 2016) in two approaches.

MR approach using individual-level data. Details on the measurement of quantitative traits and the definitions of diseases in the LifeLines cohort are presented elsewhere (Amini et al., 2016). We assessed the causal association of eos-GRS $\mathrm{GLs}_{\mathrm{Ls}}$ using standard MR procedures in individuallevel data of eosinophil count and 20 outcomes data available in the LifeLines cohort study, while adjusted for age and gender (Abbasi et al., 2015). We used the two-stage, least-squares (2SLS; Abbasi et al., 2015) method (using SPSS statistical software version 22; SPSS, Inc., Chicago, USA) to generate the causal estimate of an elevated eosinophil count on change of intermediate trait levels and risk of diseases. The first stage of the 2SLS method is to examine the 


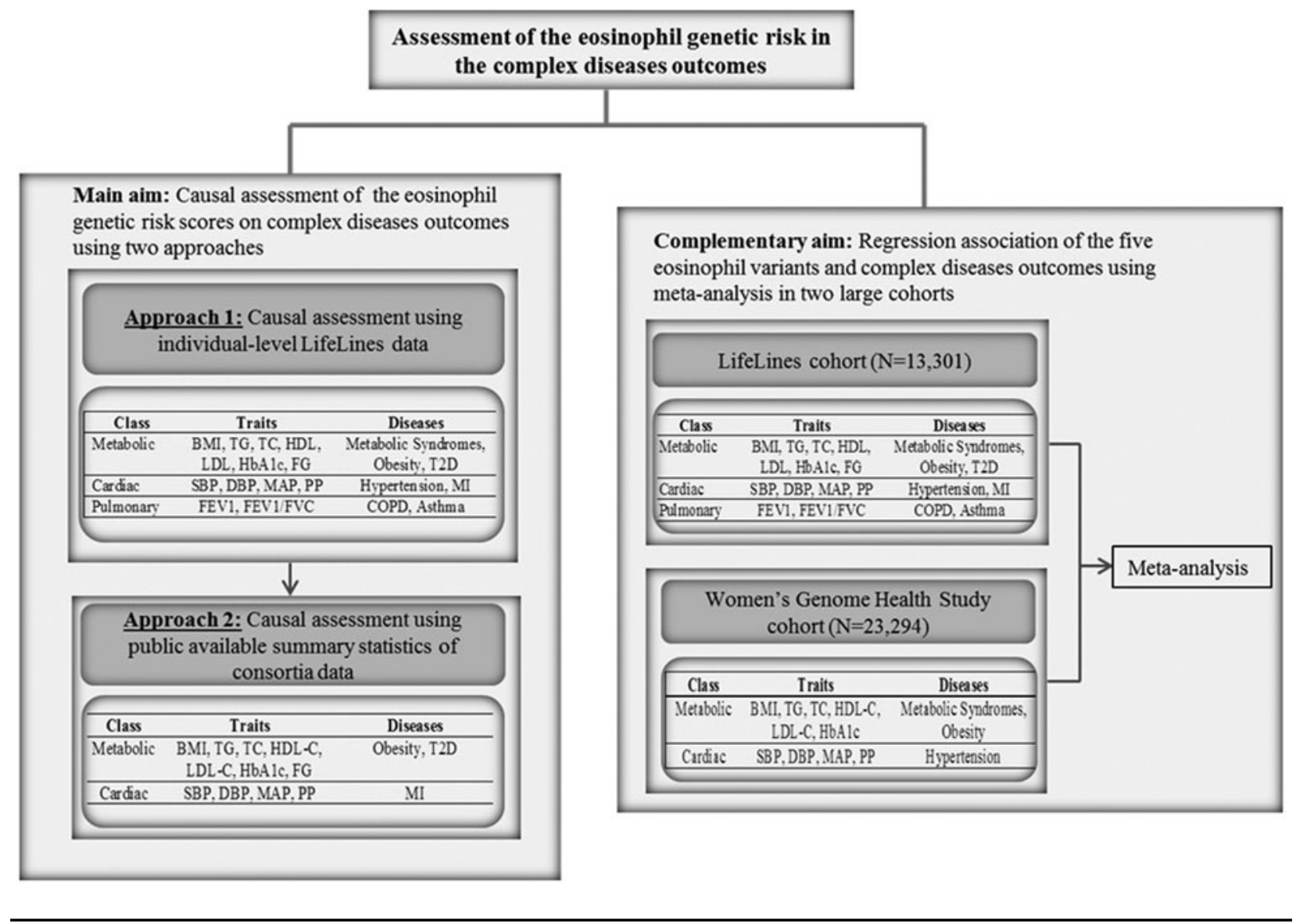

\section{FIGURE 1}

Flow chart of the study design. $\mathrm{BMI}=$ body mass index, $\mathrm{TG}=$ triglycerides, $\mathrm{TC}=$ total cholesterol, $\mathrm{HDL}=$ high density lipoprotein, $\mathrm{LDL}=$ low density lipoprotein, HbA1c = hemoglobin A1c, FG = fasting glucose, SBP = systolic blood pressure, DBP = diastolic blood pressure, $\mathrm{MAP}=$ mean arterial pressure, $\mathrm{PP}=$ pulse pressure, $\mathrm{HTN}=$ hypertension, $\mathrm{MI}=$ myocardial infarction, $\mathrm{FEV}_{1}=$ Forced expiratory volume in one second, FVC = forced vital capacity, COPD = chronic obstructive pulmonary diseases.

observational association between eos-GRS $\mathrm{LLs}_{\mathrm{Ls}}$ and eosinophil count by means of linear regression and saving the predicted values. In the second stage, the predicted values of eosinophil count from the first stage are used as covariates, with traits or diseases as dependent variable in a linear or logistic regression model (Abbasi et al., 2015). The causal estimate is this second-stage regression coefficient for the change in the complex outcomes caused by a 1-SD change in the eosinophil count (Burgess et al., 2017). In addition, we estimated an $F$ statistic for each outcome to ensure the strength of eos-GRSs as instrumental variables using the formula: $F$ statistic $=\left[R^{2} \times(n-1-K)\right] /\left[\left(1-R^{2}\right)\right.$ $\times K]$, where ' $R^{2}$ ' represents the proportion of variance from the first-stage, ' $n$ ' represents the sample size, and ' $K$ ' represents the number of instrumental variables included in the model (i.e., for this study, $K=1$; Rice, 1995). An $F$ statistic greater than 10 indicates sufficient strength to ensure the validity of the instrument variable analysis, whereas $R^{2}$ in percent is used as a measure of the percent contribution of genotypes to the variation in eosinophil count (Burgess et al., 2013). To test for pleiotropy, we performed linear and logistic regression with eosinophil count as an independent variable and outcomes as a dependent variable, and then saved the residuals. Next, we tested the correlation between the genotype and the residuals (Li et al., 2014).

MR approach using summary statistics data. We estimated the causal association of eos-GRS $\mathrm{GWAS}_{\text {G }}$ on outcome of interest using summary statistics for each eosinophil genetic variant obtained from publicly available large consortia data of studied outcomes (Berndt et al., 2013; Coronary Artery Disease Genetics Consortium et al., 2011; Dupuis et al., 2010; Global Lipids Genetics Consortium et al., 2013; Morris et al., 2012; Soranzo et al., 2009; Wain et al., 2011; Yang et al., 2012). Analyses were performed using the grs.summary module as part of the Genetics ToolboX R (version 2.15.1 for Windows; Vienna, Austria). The grs.summary module approximates the regression of an additive GRS onto a response variable or outcome. The method of analysis is described in detail elsewhere (Dastani et al., 2012; Prins et al., 2016). Since in causal inference the heterogeneity of the genetic variants' effect potentially 
indicates pleiotropic effects that violate the MR assumption (Abbasi et al., 2015; Burgess et al., 2013; Prins et al., 2016), we checked the goodness-of-fit test in the gtx package for the presence of heterogeneity. A $p<.05$ was considered statistically significant for the heterogeneity. When significant heterogeneity was detected, genetic variants that contributed most to the statistical heterogeneity were iteratively removed until the heterogeneity test was no longer significant, and genetic risk score analyses were repeated for the remaining subset of genetic variants (Dastani et al., 2012). Since the ln-transformed eosinophil count was used as the response variable in the reference study (Gudbjartsson et al., 2009), to obtain the effect sizes for each of the eosinophil genetic variants, a unit increase in ln-eosinophil equals a 10 symmetric percentage $(\mathrm{s} \%)$ increase in actual eosinophil count. This corresponds to a unit change in levels of a continuous outcome or logit of risk-estimate for a dichotomous outcome (Cole, 2000). Therefore, the causal estimate of instrumental variable was presented for each outcome as corresponding to a $10-\mathrm{s} \%$ increase in actual eosinophil count (Rice, 1995).

A $p<.05$ was taken as nominally significant, and to address multiple testing, Bonferroni corrected $p$ values were considered as statistically significant with $p<2.5 \times 10^{-3}$ $(0.05 / 20$ total analyses) for individual-level data analyses and $p<3.6 \times 10^{-3}(0.05 / 14$ total analyses $)$ for summary statistics analyses.

\section{Results}

Details of the study population characteristics are presented in Supplementary Results.

\section{Individual Variants Results}

Using the individual-level LifeLines data $(n=13,301)$, we replicated significant associations of $0.09 S D$ units increase of eosinophil count with an increase of one copy of SH2B3-rs3184504*T $\left(p=3.9 \times 10^{-13}\right), 0.04$ SD units with IL5-rs4143832* G $\left(p=1.3 \times 10^{-3}\right), 0.03$ SD units with $I K Z F 2-\mathrm{rs} 12619285^{*} \mathrm{G}\left(p=1.5 \times 10^{-3}\right), 0.09 S D$ units with GATA2-rs4857855* $\mathrm{C}\left(p=1.4 \times 10^{-8}\right)$, and $0.06 S D$ units with $I L 1 R L 1-\mathrm{rs} 1420101^{*} \mathrm{~A}\left(p=1.0 \times 10^{-4}\right)$ variants (Figure S1). We also found that SH2B3-rs3184504*T explained $0.30 \%$ variance of eosinophil count and this was $0.05 \%$ for $I L 5$-rs $4143832 * \mathrm{G}, 0.001 \%$ for IKZF2rs $12619285^{*} \mathrm{G}, \quad 0.20 \%$ for GATA2-rs $4857855^{*} \mathrm{C}, 0.06 \%$ for IL1RL1-rs1420101*A. eos-GRS ${ }_{\mathrm{LLs}}$ explained $0.60 \%$ of eosinophil count variance.

In the sensitivity analysis, we assessed the association of the five eosinophil genetic variants with metabolic, cardiac, and pulmonary intermediate traits and diseases using metaanalysis in two large cohorts of LifeLines and the Womens's Genome Health Study (WGHS) with a total sample size of 36,595 individuals (Supplementary Methods and Supplementary Results). The results in the metabolic class showed that an increase in one copy of GATA2-rs4857855* $\mathrm{C}$ was significantly associated $\left(p<2.5 \times 10^{-3}\right)$ with higher TG and SH2B3-rs3184504*T with lower LDL-C. Additionally, an increase in one copy of SH2B3-rs3184504*T was nominally $(p<.05)$ associated with a higher HbA1c level. The results on metabolic diseases indicated a nominal significant association of IL5-rs $4143832 * \mathrm{G}$ with lower risk of obesity. In the cardiac class, we found a significant association of SH2B3-rs3184504*T with higher SBP, DBP, and MAP values. In the pulmonary class, there was a nominal significant association of $S H 2 B 3-r s 3184504 * \mathrm{~T}$ with lower $\mathrm{FEV}_{1} / \mathrm{FVC}$. We also found nominal significant associations of GATA2rs $4857855^{*} \mathrm{C}$ with lower risk of COPD and asthma, IKZF2rs $12619285^{*} \mathrm{G}$ and IL1RL1-rs1420101*A with higher risk of asthma. Details on effects and odds ratio $(O R)$ of metaanalysis results are presented in Supplementary Results and Figures S2 and S3.

\section{GRS MR Results}

We performed MR analyses using two approaches to distinguish the potential causal associations between eosinophil count and three classes of metabolic, cardiac, and pulmonary outcomes. As the eosinophil count data was not available in the WGHS, MR analysis in individual-level data only has been performed in the LifeLines cohort study.

Figure 2 presents the estimated causal associations of eosinophil count on traits and diseases in the metabolic class. In the first approach, eos-GRS $\mathrm{LLs}_{\mathrm{L}}$ showed no significant causal association with metabolic intermediate traits and diseases (Figure 2a.1 and a.2). The $F$ statistics derived from individual-level LifeLines data for metabolic traits were $>63.3$; and for diseases, except for T2D $(=3.8)$, they were $>13.4$. In the second approach, the eos-GRS $\mathrm{GWAS}_{\mathrm{GW}}$ showed that a 10 -s\% higher genetically determined eosinophil count had a significant association with a $0.10\left( \pm S E 0.03 ; p=3.9 \times 10^{-4}\right) \mathrm{mmol} / \mathrm{L}$ higher TC, $0.13\left( \pm 0.03 ; p=4.5 \times 10^{-6}\right) \mathrm{mmol} / \mathrm{L}$ higher HDLC, $0.09\left( \pm 0.03 ; p=3.8 \times 10^{-3}\right) \mathrm{mmol} / \mathrm{L}$ higher LDL-C (Figure 2b.1). However, there was significant evidence of heterogeneity of effect sizes among the five constituting genetic variants from meta-analyses results (Figure S4). We performed stepwise removal of genetic variants from

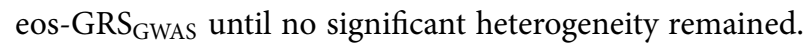
This adjustment resulted in the removal of the SH2B3rs $3184504^{*} \mathrm{~T}$ genetic variant. Consequently, the significant association of eosinophil count with TC $(0.09 \pm 0.05$, $p=0.07)$, HDL-C $(0.05 \pm 0.04, p=.21)$, and LDL-C $(0.09 \pm 0.04, p=.06)$ changed to non-significant, while the direction of effect remained consistent. In addition, eos-GRS $\mathrm{GWAS}_{\mathrm{GW}}$, as an instrumental variable, showed that a genetically $10-s \%$ increase in eosinophil count was significantly associated with lower odds of obesity (OR 0.81 , $95 \%$ confidence interval (CI) $[0.74,0.89] ; p=5.0 \times 10^{-6}$; Figure 2 b.2), while no heterogeneity was found in the 


\section{LifeLines metabolic outcomes}

2a.1

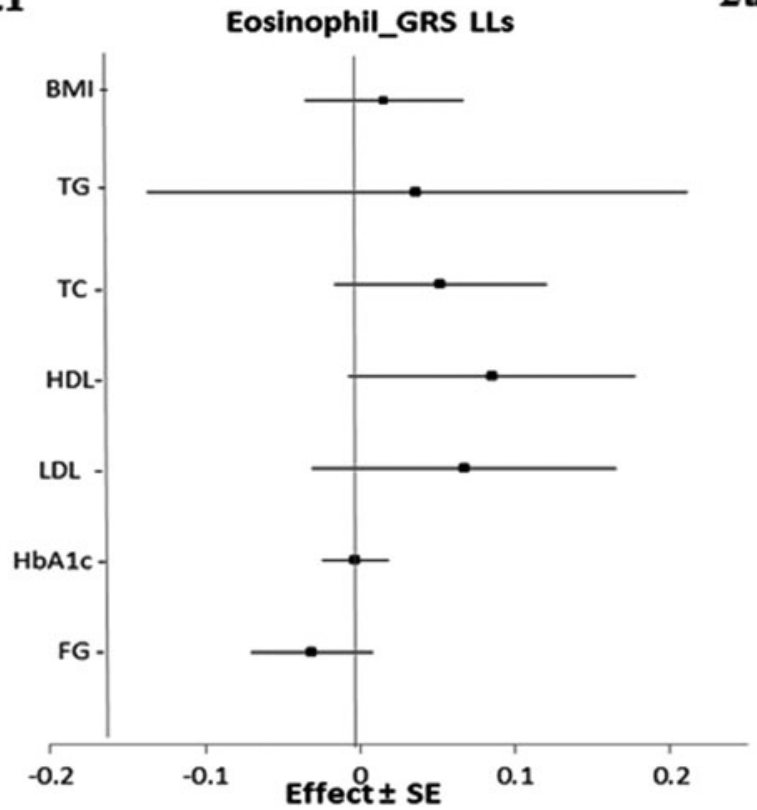

$2 \mathrm{a.2}$

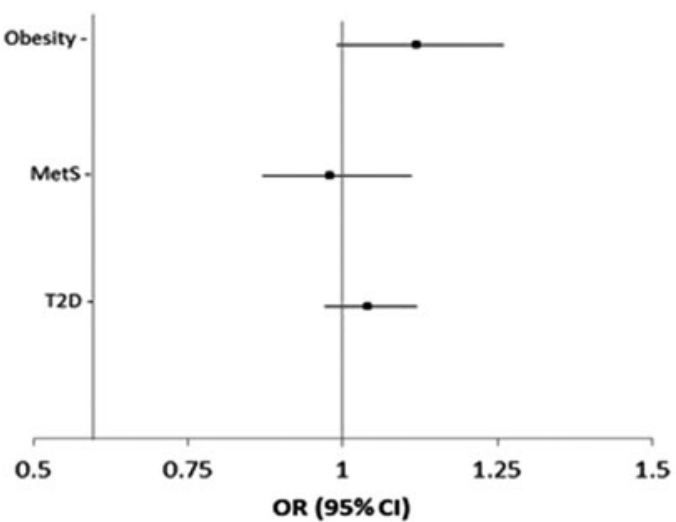

GWAS consortia metabolic outcomes

2b.1

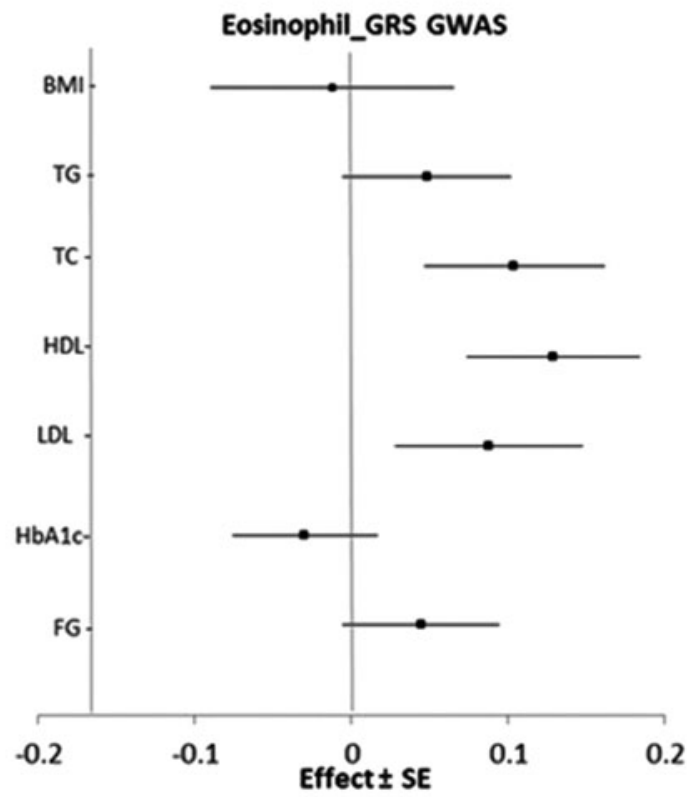

\section{$2 \mathbf{b} .2$}

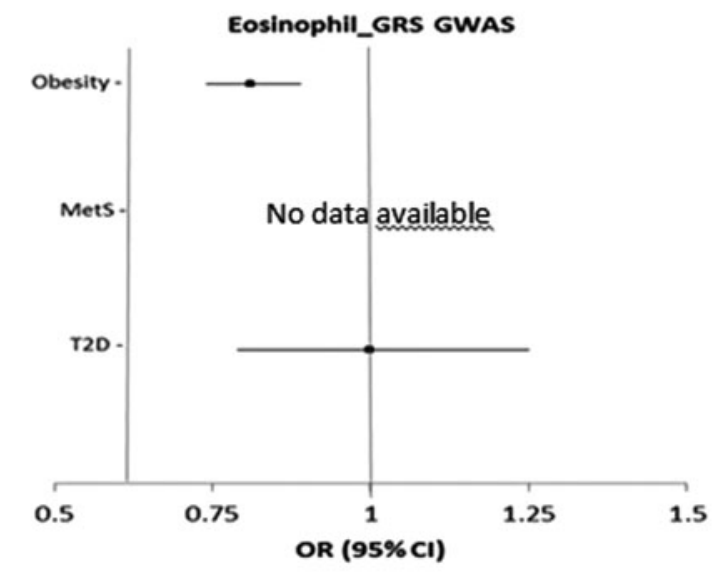

\section{FIGURE 2}

The causal effects with standard error of the eosinophil genetic risk scores on quantitative traits and the odds ratio with $95 \%$ confidence intervals on diseases in metabolic class, derived from the individual-level LLs data (eos-GRS $\mathrm{LLs}_{1}$ a.1, a.2) and the GWAS consortia (eosGRS $_{\text {GWAs; }}$ b.1 and b.2) before genetic variants heterogeneity effect adjustment. The causal estimate of the instrumental variable, which is presented for each outcome, corresponds to a $10-s \%$ increase in actual eosinophil count. No GWAS consortium data was available for metabolic syndrome disease. GRS = genetic risk score, LLs = LifeLines cohort study, GWAS = genome wide association study, SE = standard error, $\mathrm{OR}=$ odds ratio, $\mathrm{Cl}=$ confidence interval, $\mathrm{BMI}=$ body mass index, $\mathrm{TG}=$ triglycerides, $\mathrm{TC}=$ total cholesterol, $\mathrm{HDL}=$ high density lipoprotein, LDL = low density lipoprotein, HbA1c = hemoglobin A1c, FG = fasting glucose. 


\section{LifeLines cardiac outcomes}
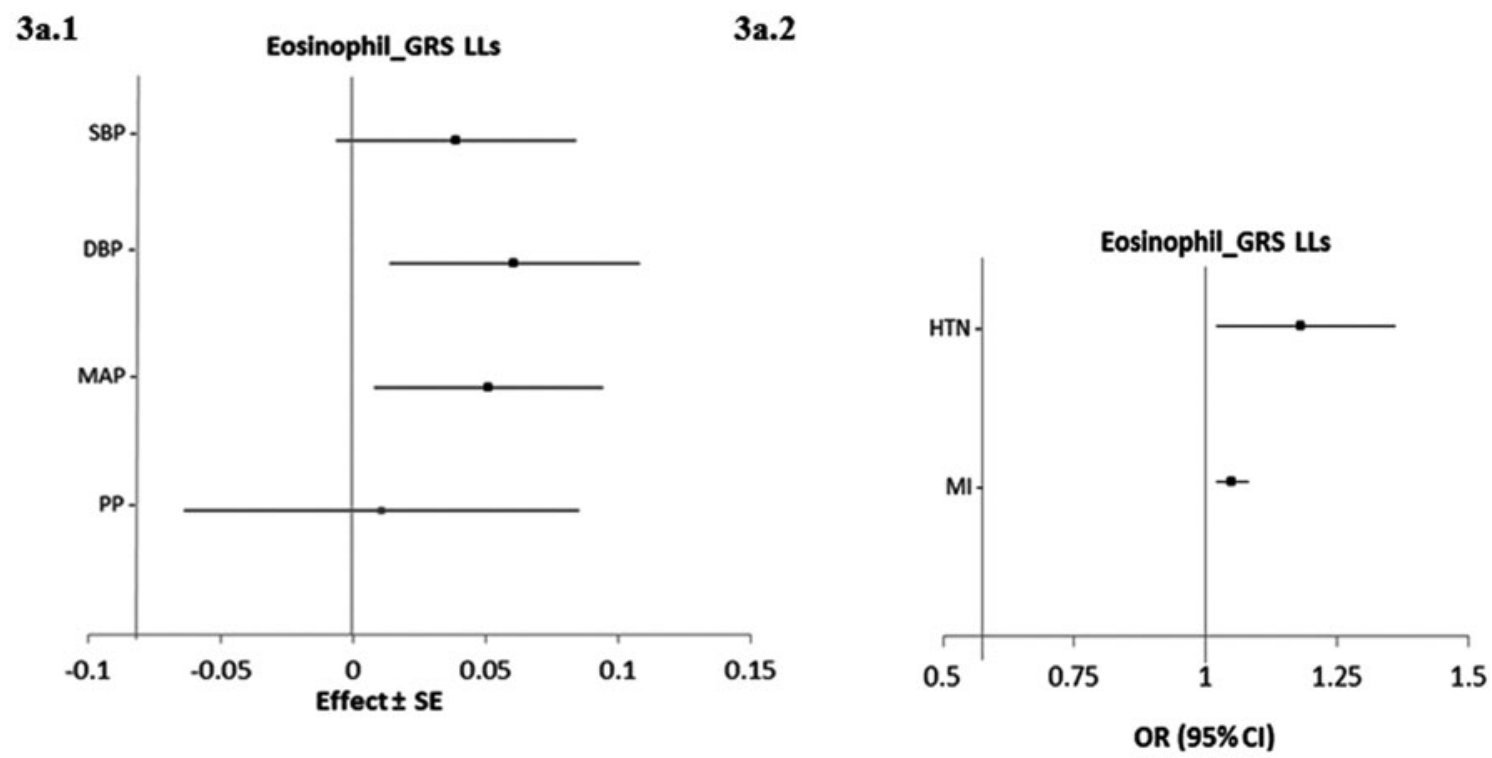

GWAS consortia cardiac outcomes

3b.1

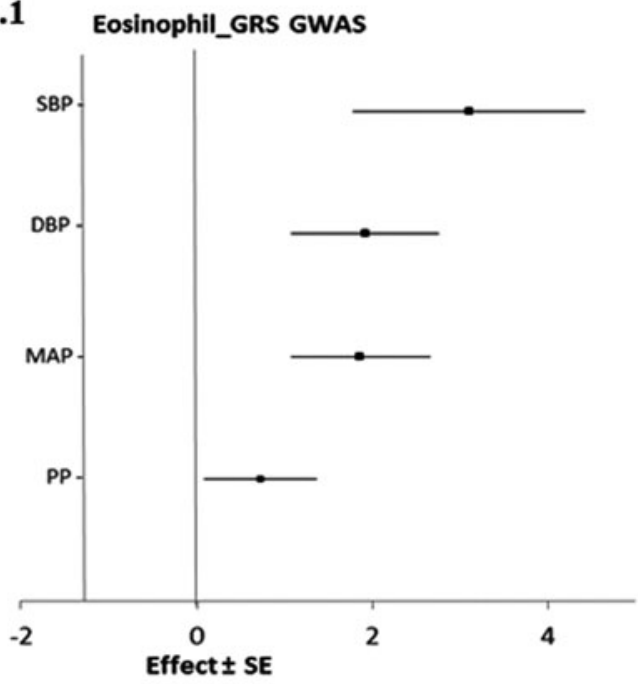

$3 \mathbf{b . 2}$

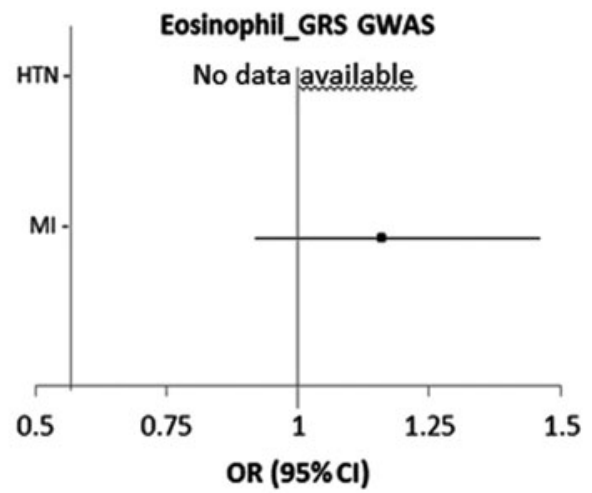

\section{FIGURE 3}

The causal effects with standard error of the eosinophil genetic risk scores on quantitative traits and the odds ratio with $95 \%$ confidence intervals on diseases in cardiac class, derived from the individual-level LLs data (eos-GRS ${ }_{\mathrm{LL}} ;$ a.1 and a.2) and the GWAS consortia (eosGRS $_{\text {GWAS; }}$ b.1 and b.2) before genetic variants heterogeneity effect adjustment. The causal estimate of the instrumental variable, which is presented for each outcome, corresponds to a 10-s\% increase in actual eosinophil count. No GWAS consortium data was available for hypertension disease. GRS = genetic risk score, LLs = LifeLines cohort study, GWAS = genome wide association study, SE = standard error, $\mathrm{OR}=$ odds ratio, $\mathrm{Cl}=$ confidence interval, $\mathrm{SBP}=$ systolic blood pressure, $\mathrm{DBP}=$ diastolic blood pressure, $\mathrm{MAP}=$ mean arterial pressure, $\mathrm{PP}=$ pulse pressure, $\mathrm{HTN}=$ hypertension, $\mathrm{MI}=$ myocardial infarction.

analyses. No significant causal association was found between eos-GRS $\mathrm{GWAS}_{\mathrm{S}}$ and the other studied metabolic traits or diseases (Figure $2 \mathrm{~b} .1$ and b.2).

Figure 3 shows the estimated causal associations of eosinophil count on traits and diseases in the cardiac class. In the first approach, eos-GRS $\mathrm{LLs}_{\text {s }}$ provided nominal significant evidence of associations between eosinophil count and blood pressure measures-an increase of $0.06( \pm 0.02 ; p=$ $.010) \mathrm{mmHg}$ in DBP and $0.05( \pm 0.02 ; p=.021) \mathrm{mmHg}$ in MAP per 1-SD higher eosinophil count (Figure 3a.1). However, the pleiotropic effect was significant $\left(p_{\text {pleiotropy }}<\right.$ .05 ) for both traits. eos-GRS LLs $_{\text {showed no significant causal }}$ association with the other quantitative traits in the cardiac class (Figure 3a.1). eos-GRS LLs $_{\text {showed moderate but }}$ 
4a.1

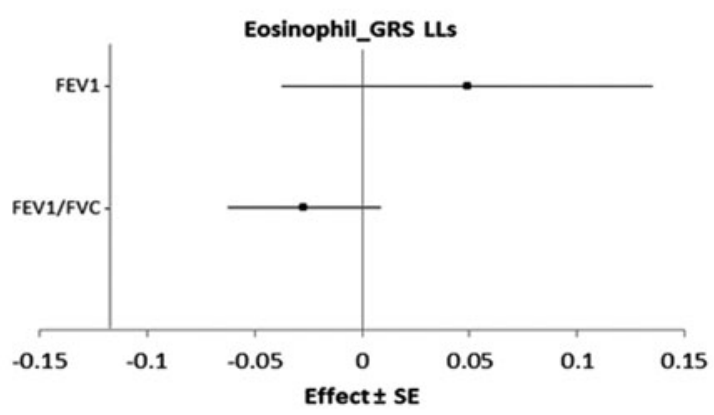

LifeLines pulmonary outcomes

$4 a .2$

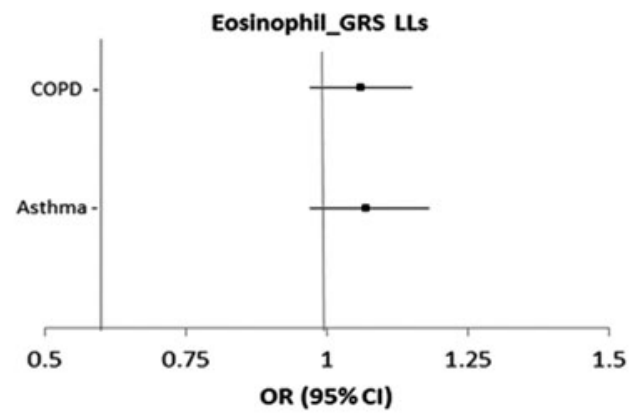

\section{FIGURE 4}

The causal effect with standard error of the eosinophil genetic risk scores on quantitative traits and the odds ratio with $95 \%$ confidence

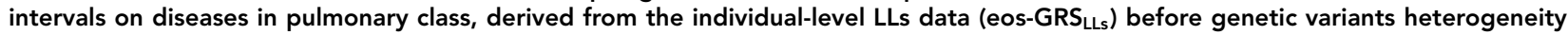
effect adjustment. The causal estimate of the instrumental variable, which is presented for each outcome, corresponds to a 10 -s\% increase in actual eosinophil count. GRS = genetic risk score, LLs = LifeLines cohort study, $\mathrm{SE}=$ standard error, OR $=$ odds ratio, $\mathrm{Cl}=$ confidence interval, $\mathrm{FEV}_{1}=$ Forced expiratory volume in one second, FVC = forced vital capacity, COPD = chronic obstructive pulmonary diseases.

nominal significant effect on the risk of HTN $(O R 1.18,95 \%$ CI $[1.02,1.36] ; p=.04)$ and MI (OR 1.05, 95\% CI [1.02, $1.08] ; p=.03$; Figure 3a.2) with $F$ statistics equal to 71.2 for cardiac traits, 19.3 for hypertension, and 2.0 for MI. However, the pleiotropic effect of eos-GRS $\mathrm{LLs}_{\mathrm{s}}$ for all cardiac outcomes was significant $\left(p_{\text {pleiotropy }}<.05\right)$. Stepwise removal of the potential pleiotropic SH2B3-rs3184504*T genetic variant from eos-GRS $\mathrm{LLs}_{\mathrm{Ls}}$ led to non-significant associations. When MR analysis of eos-GRS was tested using summary statistics of consortia data, the eos-GRS GWAS $_{\text {showed that a }}$ $10-s \%$ higher genetically determined eosinophil count had a significant association with a $3.10\left( \pm 0.67, p=4.01 \times 10^{-6}\right)$ $\mathrm{mmHg}$ higher SBP, $1.92\left( \pm 0.43, p=6.81 \times 10^{-6}\right) \mathrm{mmHg}$ higher DBP, and $1.86\left( \pm 0.40, p=3.83 \times 10^{-6}\right) \mathrm{mmHg}$ higher MAP (Figure 3b.1). However, there was significant evidence of heterogeneity of the effect sizes among the five constituting genetic variants (Figure S4). After removal of $\mathrm{SH} 2 \mathrm{~B} 3-\mathrm{rs} 3184504^{*} \mathrm{~T}$ as a heterogenic genetic variant, the significant association of SBP $(1.38 \pm 0.08 ; p=$ $.08)$, DBP $(0.32 \pm 0.49 ; p=.52)$ and MAP (0.67 \pm 0.47 ; $p=.15)$ changed to non-significant. eos-GRS $\mathrm{GWAS}_{\mathrm{G}}$ provided no significant causal association on $\mathrm{MI}$, and for hypertension diseases, no consortium data was available (Figure 3b.2).

Figure 4 shows the causal associations between eosinophil count and traits and diseases in the pulmonary class in individual-level LifeLines data. As the consortium data on pulmonary traits and diseases was not publicly available, the pulmonary traits and diseases were only tested for causal association using eos-GRS $\mathrm{GLs}_{\mathrm{Ls}}$ eos-GRS $\mathrm{GLs}_{\mathrm{L}}$ showed no statistically significant causal association for these traits and diseases (Figure 4a.1 and a.2). The derived $F$ statistics were $>72.5$ for pulmonary traits, 7.4 for COPD, and 6.2 for asthma diseases.

\section{Discussion}

In our study, we replicated the significant associations between five eosinophil variants and eosinophil count. Additionally, we found significant associations between eosinophil genetic risk variants and TG, LDL-C, SBP, DBP, and MAP. Then, we used MR analyses to investigate the causal association between eosinophil count and a wide range of metabolic, cardiac, and pulmonary intermediate traits and diseases. We combined five eosinophil genetic variants into eos-GRS as instrumental variable in order to do MR analyses using individual-level LifeLines data and summary statistics of the consortia data. We found a protective causal association between eosinophil count and obesity using summary statistics, but this was inconclusive in the individual-level LifeLines data. We found no statistically significant causal association between eosinophil count and the other studied traits or diseases using eos-GRS $\mathrm{LLs}_{\mathrm{L}}$ and eos-GRS $\mathrm{GWAS}_{\mathrm{S}}$ after correction for heterogeneity through removal of potentially pleiotropic genetic variants. This suggests that the associations of blood eosinophil count and the studied complex traits and diseases may not be causal.

As a pleiotropic multifunctional leukocyte, eosinophil is important for the initiation and progression of diverse inflammatory responses (Jacobsen et al., 2011) that are implicated in the pathophysiology of common complex diseases (Amini et al., 2016; Bafadhel et al., 2012; Fukui et al., 2009; Kim, Noh et al., 2008; Shim et al., 2006). In observational studies, the inconsistent findings of correlation between eosinophil count and complex outcomes (Amini et al., 2016; Gkrania-Klotsas et al., 2010; Kim, Choi et al., 2006; Kim, Noh et al., 2008; Meng et al., 2012; Shim et al., 2006) might be due to the presence of measured and unmeasured confounders (Davey Smith \& Ebrahim, 2003, 2005). In MR, a well-known statistical method for causality 
assessment, it is assumed that the genetic variation modifying the exposure should also affect the risk of outcome if the exposure is causally involved in the pathogenesis of outcome (Burgess et al., 2017). As genes randomly allocate at conception, their effects on exposures are unaffected by classical confounding factors and reverse causation (Davey Smith \& Ebrahim, 2003) or systemic errors would be largely prevented compared to an ordinary linear regression analyses (Davey Smith \& Ebrahim, 2003, 2005). In a previous GWA study, Gudbijartson et al. (2009) reported that five genetic variants (SH2B3-rs3184504, IL5-rs4143832, IKZF2rs12619285, GATA2-rs4857855, and IL1RL1-rs1420101) were associated with eosinophil count in European and Asian populations. In this study, we replicated those associations between all five genetic variants and eosinophil count, with comparable effect sizes and the same direction of effect compared to the finding from the study of Gudbjartson et al.. Thus, as the starting point of our analysis, we used well-replicated short nucleotide polymorphisms (SNPs) robustly associated with eosinophil count. However, the explained variance of blood eosinophil count was limited (ranging from $0.001 \%$ to $0.3 \%$ from individual genetic variants and $0.6 \%$ from total genetic risk score).

Several observational studies indicated that a higher eosinophil count was associated with a higher risk of metabolic disorders (Amini et al., 2016; Babio et al., 2013; Kim, Choi et al., 2006; Meng et al., 2012; Shim et al., 2006). In our study, using eos-GRS $\mathrm{GWAS}_{\text {, we found a significant }}$ causal association between a 10 -s\% increase in eosinophil count and a 1.23-fold decrease in the risk of obesity. However, using the individual-level data from the LifeLines cohort study, we did not find this causal association with obesity. In the individual-level data analysis, we used a twostage method, which is efficient but can be biased with a weak instrument; in the summary data analysis, however, the likelihood-based method has been used, which is asymptotically efficient and less biased (Burgess et al., 2017). In the summary data analysis approach, information about genetic variants in eosinophil count (Gudbjartsson et al., 2009) and metabolic outcomes (Berndt et al., 2013; Dupuis et al., 2010; Global Lipids Genetics Consortium et al., 2013; Morris et al., 2012; Yang et al., 2012) came from different sources of European ancestry, but it assumes that the two samples were from comparable populations in terms of genomic ancestry (Burgess et al., 2015); therefore, ethnic difference could not have affected our results. But, when we compared the odds estimates from our instrumental variable analysis $(0.81)$ with the ordinary linear regression analysis (Amini et al., 2016; 1.18), we found no concordant estimates in obese individuals (Amini et al., 2016; Kim, Noh et al., 2008; Meng et al., 2012). Taken together, it remains inconclusive whether eosinophil count is indeed a causal protective factor for obesity, as no other previous biologically plausible reason has been reported for such a causal association. Therefore, it is highly unlikely that they have true causal effects. Using eos-GRSs from individual-level LifeLines data and consortia data, we found no significant causal association with any of the other investigated quantitative traits and diseases in the metabolic class. In the study by Astle et al. (2016), they also could not find any causal association between eosinophil and cardiometabolic outcomes in spite of their larger sample size. In sensitivity analysis using individual genetic variants, we found a significant association of GATA2-rs4857855 and higher TG. Also, SH2B3-rs3184504 showed significant association with lower LDL-C, which was consistent with results from Talmud et al. (2009), while in other studies this variant showed either a significant association with higher LDL-C (Aghabozorg Afjeh et al., 2014) or no significant association with LDL-C level at all (Gudbjartsson et al., 2009). Thus, current evidence does not support a causal role of eosinophil count for metabolic outcomes.

In the cardiac class, using eos-GRSs in both approaches, there was a significant causal association between eosinophil count and SBP, DBP, and MAP measurements; however, these associations were largely influenced by the SH2B3-rs3184504 variant. A few epidemiological studies showed inconsistent results on the association between eosinophil count and blood pressure (Amini et al., 2016; Kim, Noh et al., 2008; Meng et al., 2012). We also could not quantify any significant causal association between eosinophil count and cardiac outcomes. This result is in line with Astle et al.'s (2016) study, which did not find causal associations between blood cell indices, including eosinophil and cardiovascular diseases (Astle et al., 2016), using a large sample size. In our study, SH2B3rs3184504 showed a significant association with SBP, DBP, and MAP, which was consistent with results of the International Consortium for Blood Pressure Genome-Wide Association Studies et al. (2011). Other studies indicated that this genetic variant was also significantly associated with other blood cell traits (Tin et al., 2013; van der Harst et al., 2012), blood platelet numbers (Soranzo et al., 2009), hemoglobin concentration (Ganesh et al., 2009), hematocrit (Ganesh et al., 2009), LDL-C (Talmud et al., 2009), cardiovascular risks (Gudbjartsson et al., 2009; Schunkert et al., 2011), and multiple autoimmune disorders (Alcina et al., 2010; Barrett et al., 2009; Stahl et al., 2010). The association of SH2B3-rs3184504 variant with multiple complex phenotypes points to its strong pleiotropic characteristic (Ding \& Kullo, 2011; Pickrell et al., 2009). In our study, there was also statistical evidence for a pleiotropic effect of this variant, which violates MR assumptions. In a Chinese population study, no significant associations were found between eosinophil genetic variants and chronic heart diseases (Lian et al., 2013; Ye et al., 2015). Altogether, across the different analyses (evidence from the observational study (Amini et al., 2016) and MR analyses), the results on cardiac outcomes consistently suggested an absence of causal association. 
In the pulmonary class, using eos-GRS $\mathrm{LLs}_{\mathrm{L}}$, we found no significant causal association between eosinophil count and pulmonary traits and diseases. Since consortium data on pulmonary outcomes was not available, MR analysis using summary statistics could not be performed. In individuallevel LifeLines data, the $F$ statistic values for pulmonary traits were higher than 72.5 , but for diseases the values were less than 7.4, indicating that weak instrument bias is likely to have had a substantial influence on our analyses in pulmonary diseases. Statistical power in MR analysis is a function of parameters including the proportion of variation in the exposure variable explained by the genetic predictor and the true causal association between the exposure and outcome variable. Therefore, despite the significant association between eosinophil count and pulmonary outcomes in previous observational studies (Amini et al., 2016; Hospers et al., 2000; Mensinga et al., 1992), we found no causal association of eosinophil on pulmonary diseases, which might be due to power limitations of the study. Also, in our large cohort, we showed no causal association of eosinophils with pulmonary intermediate traits $\left(\mathrm{FEV}_{1}\right.$ and $\left.\mathrm{FEV}_{1} / \mathrm{FVC}\right)$ as determinants of pulmonary diseases pathophysiology. Our results were inconsistent with a study from the United Kingdom, which indicated significant causal associations between eosinophils and asthma, and provided evidence that eosinophils are key effector cells in the pathogenesis of asthma (Astle et al., 2016). The observed causal association in that study may be explained by a larger sample size and stronger instrumental variable (Astle et al., 2016). Since patients with pulmonary diseases can be clinically or physiologically stratified into several subgroups (Fahy, 2015), the lack of causal associations in our study might be due to biases by subgroup stratification or possibly insufficient power to identify a relatively small causal effect of eosinophil on pulmonary outcomes in our database. As well, environmental factors over lifetime might mediate the potential causal association between genetically elevated eosinophil and asthma, which needs to be investigated. When we tested the association between individual eosinophil genetic variant and pulmonary outcomes, we noticed nominal associations of SH2B3rs3184504 with $\mathrm{FEV}_{1} / \mathrm{FVC}$, GATA2-rs4857855 with COPD, IKZF2-rs12619285 and IL1RL1-rs1420101 with asthma. Several studies indicated that $I L 1 R L 1$ gene cluster genetic variants, including IL1RL1-rs1420101, were associated with asthma, atopy, and the development of childhood asthma (Akhabir et al., 2014; Ali et al., 2009; Gudbjartsson et al., 2009; Reijmerink et al., 2008; Savenije et al., 2011; Torgerson et al., 2011). Consistent with our results, Tulah et al. (2013), using UK family cohort data, found no association between IL1RL1-rs1420101 and asthma and $\mathrm{FEV}_{1}$; however, in an Icelandic cohort study, this variant did show a significant association with asthma (Gudbjartsson et al., 2009). When all of the evidence is taken together, a direct link between eosinophil and pulmonary outcomes remains to be elucidated and should be interpreted with caution.

Our study has several strengths. We applied MR analyses using two models of individual-level data and summary statistics data. In individual-level LifeLines data, all study participants followed the same standardized protocols and were examined at the same clinic; thus, the measurements and laboratory assays for all subjects were comparable. For the consortia summary statistics data, the large sample size is a major advantage. However, there were potential biases to be considered in our analysis. First, the power to detect an association in population-based studies is restricted by a low prevalence of disease (Table S2); thus, the non-significant results in diseases may not warrant rejection of the role of eosinophil-associated genetic variants in the pathogenesis of complex diseases. Second, using multiple genetic variants in a GRS for MR analysis, statistical power can be increased by explaining a greater proportion of eosinophils variance. Despite this, only a small proportion $(0.6 \%)$ of variance in eosinophils was explained. Therefore, follow-up analyses are needed using a stronger eosinophil genetic risk score from forthcoming studies. Third, it should be taken into account that genegene and gene-environment interaction effects probably contribute to inter-individual variation and the causal effect size of this hematologic trait. Fourth, for many complex diseases, the disease is not a single disease but rather a syndrome consisted of many heterogeneous underlying molecular mechanisms. For example, both asthma and COPD include eosinophilic and non-eosinophilic types. As the importance of eosinophil as a causative factor is really dependent on which population to study, follow-up studies may need to focus on a stratified population in these complex diseases.

\section{Conclusion}

Following the observational association of eosinophil count with complex outcomes, we found that eosinophil genetic variants were significantly associated with TG, LDL-C, SBP, DBP, and MAP. However, the causal role of eosinophil on complex metabolic, cardiac, and pulmonary outcomes could not be verified. The causality of eosinophils in the pathogenesis of asthma needs to be investigated in further studies with stronger instrumental variables using a larger number of eosinophil count-associated SNP sets. Rather than being a causal effect, our results suggest that the widespread associations of eosinophil count with complex disease outcomes in observational studies may at least partly be caused by reverse causation and pleiotropic effects.

\section{Acknowledgments}

The authors would like to acknowledge the services of the Lifelines Cohort Study, the contributing research centers delivering data to Lifelines, the staff from the LifeLines 
Cohort Study, and the Medical Biobank Northern Netherlands, the participating general practitioners and pharmacists, and all the study participants.

The LifeLines Cohort Study is supported by the Netherlands Organization of Scientific Research NWO (grant 175.010.2007.006), the Economic Structure Enhancing Fund (FES) of the Dutch government, the Ministry of Economic Affairs, the Ministry of Education, Culture and Science, the Ministry for Health, Welfare and Sports, the Northern Netherlands Collaboration of Provinces (SNN), the Province of Groningen, University Medical Center Groningen, the University of Groningen, Dutch Kidney Foundation, and Dutch Diabetes Research Foundation.

The Women's Genome Health Study is supported by HL043851 and HL080467 from the National Heart, Lung, and Blood Institute and CA047988 from the National Cancer Institute, the Donald W. Reynolds Foundation and the Fondation Leducq, with collaborative scientific support and funding for genotyping provided by Amgen.

Mrs M. Amini was supported by Groningen University Institute for Drug Exploration (GUIDE), UMCG, Groningen, the Netherlands, and Digestive Disease Research Institute (DDRI), TUMS, Tehran, Iran.

\section{Disclosure of Interests}

None.

\section{Supplementary material}

To view supplementary material for this article, please visit https://doi.org/10.1017/thg.2018.6

\section{References}

Abbasi, A., Deetman, P. E., Corpeleijn, E., Gansevoort, R. T., Gans, R. O., Hillege, H. L., ... Bakker, S. J. (2015). Bilirubin as a potential causal factor in type 2 diabetes risk: A Mendelian randomization study. Diabetes, 64, 1459-1469.

Aghabozorg Afjeh, S. S., Ghaderian, S. M., Mirfakhraie, R., Piryaei, M., \& Zaim Kohan, H. (2014). Association study of rs3184504 C>T polymorphism in patients with coronary artery disease. International Journal of Molecular and Cellular Medicine, 3, 157-165.

Akhabir, L., Berube, J. C., Bosse, Y., Laviolette, M., Hao, K., Nickle, D. C., ... Sandford, A. J. (2014). Lung expression quantitative trait loci data set identifies important functional polymorphisms in the asthma-associated IL1RL1 region. The Journal of Allergy and Clinical Immunology, 134, 729-731.

Alcina, A., Vandenbroeck, K., Otaegui, D., Saiz, A., Gonzalez, J. R., Fernandez, O., ... Matesanz, F. (2010). The autoimmune disease-associated KIF5A, CD226 and SH2B3 gene variants confer susceptibility for multiple sclerosis. Genes and Immunity, 11, 439-445.

Ali, M., Zhang, G., Thomas, W. R., McLean, C. J., Bizzintino, J. A., Laing, I. A., ... Hayden, C. M. (2009). Investigations into the role of ST2 in acute asthma in children. Tissue Antigens, 73, 206-212.

Amini, M., Bashirova, D., Prins, B. P., Corpeleijn, E., LifeLines Cohort Study, Bruinenberg, M., ... Alizadeh, B. Z. (2016). Eosinophil count is a common factor for complex metabolic and pulmonary traits and diseases: The LifeLines cohort study. PLoS One, 11, e0168480.

Astle, W. J., Elding, H., Jiang, T., Allen, D., Ruklisa, D., Mann, A. L., ... Soranzo, N. (2016). The allelic landscape of human blood cell trait variation and links to common complex disease. Cell, 167, 1415-1429.e19.

Babio, N., Ibarrola-Jurado, N., Bullo, M., Martinez-Gonzalez, M., Warnberg, J., Salaverria, I., ... PREDIMED Study Investigators. (2013). White blood cell counts as risk markers of developing metabolic syndrome and its components in the PREDIMED study. PLoS One, 8, e58354-e58354.

Bafadhel, M., McKenna, S., Terry, S., Mistry, V., Pancholi, M., Venge, P., ... Brightling, C. E. (2012). Blood eosinophils to direct corticosteroid treatment of exacerbations of chronic obstructive pulmonary disease: A randomized placebocontrolled trial. American Journal of Respiratory and Critical Care Medicine, 186, 48-55.

Barrett, J. C., Clayton, D. G., Concannon, P., Akolkar, B., Cooper, J. D., Erlich, H. A., ... Type 1 Diabetes Genetics Consortium. (2009). Genome-wide association study and meta-analysis find that over 40 loci affect risk of type 1 diabetes. Nature Genetics, 41, 703-707.

Berndt, S. I., Gustafsson, S., Magi, R., Ganna, A., Wheeler, E., Feitosa, M. F., ... Ingelsson, E. (2013). Genome-wide metaanalysis identifies 11 new loci for anthropometric traits and provides insights into genetic architecture. Nature Genetics, 45, 501-512.

Burgess, S., Butterworth, A., \& Thompson, S. G. (2013). Mendelian randomization analysis with multiple genetic variants using summarized data. Genetic Epidemiology, 37, 658-665.

Burgess, S., Scott, R. A., Timpson, N. J., Davey Smith, G., Thompson, S. G., \& EPIC-InterAct Consortium. (2015). Using published data in Mendelian randomization: A blueprint for efficient identification of causal risk factors. European Journal of Epidemiology, 30, 543-552.

Burgess, S., Small, D. S., \& Thompson, S. G. (2017). A review of instrumental variable estimators for Mendelian randomization. Statistical Methods in Medical Research, 26, 23332355.

Cole, T. J. (2000). Sympercents: Symmetric percentage differences on the $100 \log (\mathrm{e})$ scale simplify the presentation of log transformed data. Statistics in Medicine, 19, 3109-3125.

Coronary Artery Disease (C4D) Genetics Consortium, Peden, J. F., Hopewell, J. C., Saleheen, D., Chambers, J. C., Hager, J., ... Collins, R. (2011). A genome-wide association study in europeans and south asians identifies five new loci for coronary artery disease. Nature Genetics, 43, 339-344.

Dastani, Z., Hivert, M. F., Timpson, N., Perry, J. R., Yuan, X., Scott, R. A., ... Kathiresan, S. (2012). Novel loci for adiponectin levels and their influence on type 2 diabetes and metabolic traits: A multi-ethnic meta-analysis of 45,891 individuals. PLoS Genetics, 8, e1002607. 
Davey Smith, G., \& Ebrahim, S. (2003). 'Mendelian randomization': Can genetic epidemiology contribute to understanding environmental determinants of disease? International Journal of Epidemiology, 32, 1-22.

Davey Smith, G., \& Ebrahim, S. (2005). What can Mendelian randomisation tell us about modifiable behavioural and environmental exposures? BMJ, 330, 1076-1079.

Davey Smith, G., \& Hemani, G. (2014). Mendelian randomization: Genetic anchors for causal inference in epidemiological studies. Human Molecular Genetics, 23, R89-R98.

Didelez, V., \& Sheehan, N. (2007). Mendelian randomization as an instrumental variable approach to causal inference. Statistical Methods in Medical Research, 16, 309-330.

Ding, K., \& Kullo, I. J. (2011). Geographic differences in allele frequencies of susceptibility SNPs for cardiovascular disease. BMC Medical Genetics, 12, 55.

Dupuis, J., Langenberg, C., Prokopenko, I., Saxena, R., Soranzo, N., Jackson, A. U., ... Morris, A. P. (2010). New genetic loci implicated in fasting glucose homeostasis and their impact on type 2 diabetes risk. Nature Genetic, 42, 105-116.

Fahy, J. V. (2015). Type 2 inflammation in asthma - Present in most, absent in many. Nature Reviews Immunology, 15, 57-65.

Fukui, M., Tanaka, M., Hamaguchi, M., Senmaru, T., Sakabe, K., Shiraishi, E., ... Nakamura, N. (2009). Eosinophil count is positively correlated with albumin excretion rate in men with type 2 diabetes. Clinical Journal of the American Society of Nephrology, 4, 1761-1765.

Ganesh, S. K., Zakai, N. A., van Rooij, F. J., Soranzo, N., Smith, A. V., Nalls, M. A., ... Lin, J. P. (2009). Multiple loci influence erythrocyte phenotypes in the CHARGE consortium. Nature Genetics, 41, 1191-1198.

Gkrania-Klotsas, E., Ye, Z., Cooper, A. J., Sharp, S. J., Luben, R., Biggs, M. L., ... Langenberg, C. (2010). Differential white blood cell count and type 2 diabetes: Systematic review and meta-analysis of cross-sectional and prospective studies. PLoS One, 5, e13405-e13405.

Global Lipids Genetics Consortium, Willer, C. J., Schmidt, E. M., Sengupta, S., Peloso, G. M., Gustafsson, S., ... Abecasis, G. R. (2013). Discovery and refinement of loci associated with lipid levels. Nature Genetics, 45, 1274-1283.

Gudbjartsson, D. F., Bjornsdottir, U. S., Halapi, E., Helgadottir, A., Sulem, P., Jonsdottir, G. M., ... Stefansson, K. (2009). Sequence variants affecting eosinophil numbers associate with asthma and myocardial infarction. Nature Genetics, 4, 342-347.

Hospers, J. J., Schouten, J. P., Weiss, S. T., Postma, D. S., \& Rijcken, B. (2000). Eosinophilia is associated with increased all-cause mortality after a follow-up of 30 years in a general population sample. Epidemiology, 11, 261-268.

International Consortium for Blood Pressure Genome-Wide Association Studies, Ehret, G. B., Munroe, P. B., Rice, K. M., Bochud, M., Johnson, A. D., ... Johnson, T. (2011). Genetic variants in novel pathways influence blood pressure and cardiovascular disease risk. Nature, 478, 103-109.
Jacobsen, E. A., Zellner, K. R., Colbert, D., Lee, N. A., \& Lee, J. J. (2011). Eosinophils regulate dendritic cells and Th2 pulmonary immune responses following allergen provocation. Journal of Immunology, 187, 6059-6068.

Kim, D. J., Noh, J. H., Lee, B. W., Choi, Y. H., Chung, J. H., Min, Y. K., ... Kim, K. W. (2008). The associations of total and differential white blood cell counts with obesity, hypertension, dyslipidemia and glucose intolerance in a Korean population. Journal of Korean Medical Science, 23, 193-198.

Kim, J. A., Choi, Y. S., Hong, J. I., Kim, S. H., Jung, H. H., \& Kim, S. M. (2006). Association of metabolic syndrome with white blood cell subtype and red blood cells. Endocrine Journal, 53, 133-139.

Li, N., van der Sijde, M. R., LifeLines Cohort Study Group, Bakker, S. J., Dullaart, R. P., van der Harst, P., .... Fu, J. (2014). Pleiotropic effects of lipid genes on plasma glucose, HbA1c, and HOMA-IR levels. Diabetes, 63, 3149-3158.

Lian, J., Huang, Y., Huang, R. S., Xu, L., Le, Y., Yang, X., ... Duan, S. (2013). Meta-analyses of four eosinophil related gene variants in coronary heart disease. Journal of Thrombosis and Thrombolysis, 36, 394-401.

Meng, W., Zhang, C., Zhang, Q., Song, X., Lin, H., Zhang, D., ... Xue, F. (2012). Association between leukocyte and metabolic syndrome in urban Han Chinese: A longitudinal cohort study. PLoS One, 7, e49875-e49875.

Mensinga, T. T., Schouten, J. P., Weiss, S. T., \& Van der Lende, R. (1992). Relationship of skin test reactivity and eosinophilia to level of pulmonary function in a community-based population study. The American Review of Respiratory Disease, 146, 638-643.

Morris, A. P., Voight, B. F., Teslovich, T. M., Ferreira, T., Segre, A. V., Steinthorsdottir, V., ... DIAbetes Genetics Replication And Meta-analysis (DIAGRAM) Consortium (2012). Large-scale association analysis provides insights into the genetic architecture and pathophysiology of type 2 diabetes. Nature Genetics, 44, 981-990.

Pickrell, J. K., Coop, G., Novembre, J., Kudaravalli, S., Li, J. Z., Absher, D., ... Pritchard, J. K. (2009). Signals of recent positive selection in a worldwide sample of human populations. Genome Research, 19, 826-837.

Prins, B. P., Abbasi, A., Wong, A., Vaez, A., Nolte, I., Franceschini, N., ... Alizadeh, B. Z. (2016). Investigating the causal relationship of C-reactive protein with 32 complex somatic and psychiatric outcomes: A large-scale cross-consortium Mendelian randomization study. PLoS Medicine, 13, e1001976.

Reijmerink, N. E., Postma, D. S., Bruinenberg, M., Nolte, I. M., Meyers, D. A., Bleecker, E. R., \& Koppelman, G. H. (2008). Association of IL1RL1, IL18R1, and IL18RAP gene cluster polymorphisms with asthma and atopy. The Journal of Allergy and Clinical Immunology, 122, 651-4.e8.

Rice, J. A. (1995). Mathematical statistics and data analysis (2nd ed.). Belmont, CA: Duxbury Press.

Savenije, O. E., Kerkhof, M., Reijmerink, N. E., Brunekreef, B., de Jongste, J. C., Smit, H. A., ... Koppelman, G. H. (2011). Interleukin-1 receptor-like 1 polymorphisms are associated with serum IL1RL1-a, eosinophils, and asthma in 
childhood. The Journal of Allergy and Clinical Immunology, 127, 750-6.e1-5.

Schunkert, H., Konig, I. R., Kathiresan, S., Reilly, M. P., Assimes, T. L., Holm, H., ... Samani, N. J. (2011). Largescale association analysis identifies 13 new susceptibility loci for coronary artery disease. Nature Genetics, 43, 333338.

Shim, W. S., Kim, H. J., Kang, E. S., Ahn, C. W., Lim, S. K., Lee, H. C., \& Cha, B. S. (2006). The association of total and differential white blood cell count with metabolic syndrome in type 2 diabetic patients. Diabetes Research and Clinical Practice, 73, 284-291.

Siva, R., Green, R. H., Brightling, C. E., Shelley, M., Hargadon, B., McKenna, S., ... Pavord, I. D. (2007). Eosinophilic airway inflammation and exacerbations of COPD: A randomised controlled trial. The European Respiratory Journal, 29, 906-913.

Soranzo, N., Spector, T. D., Mangino, M., Kuhnel, B., Rendon, A., Teumer, A., ... Gieger, C. (2009). A genome-wide metaanalysis identifies 22 loci associated with eight hematological parameters in the HaemGen consortium. Nature Genetics, 41, 1182-1190.

Stahl, E. A., Raychaudhuri, S., Remmers, E. F., Xie, G., Eyre, S., Thomson, B. P., ... Plenge, R. M. (2010). Genomewide association study meta-analysis identifies seven new rheumatoid arthritis risk loci. Nature Genetics, 42, 508-514.

Talmud, P. J., Drenos, F., Shah, S., Shah, T., Palmen, J., Verzilli, C., ... BRIGHT Consortium. (2009). Gene-centric association signals for lipids and apolipoproteins identified via the HumanCVD BeadChip. American Journal of Human Genetics, 85, 628-642.

Tin, A., Astor, B. C., Boerwinkle, E., Hoogeveen, R. C., Coresh, J., \& Kao, W. H. (2013). Genome-wide association study identified the human leukocyte antigen region as a novel locus for plasma beta-2 microglobulin. Human Genetics, 132, 619-627.

Torgerson, D. G., Ampleford, E. J., Chiu, G. Y., Gauderman, W. J., Gignoux, C. R., Graves, P. E., ... Nicolae, D. L. (2011). Meta-analysis of genome-wide association studies of asthma in ethnically diverse North American populations. Nature Genetics, 43, 887-892.

Tulah, A. S., Holloway, J. W., \& Sayers, I. (2013). Defining the contribution of SNPs identified in asthma GWAS to clinical variables in asthmatic children. BMC Medical Genetics, 14, 100 .

van der Harst, P., Zhang, W., Mateo Leach, I., Rendon, A., Verweij, N., Sehmi, J., ... Chambers, J. C. (2012). Seventyfive genetic loci influencing the human red blood cell. $\mathrm{Na}$ ture, 492, 369-375.

Wain, L. V., Verwoert, G. C., O’Reilly, P. F., Shi, G., Johnson, T., Johnson, A. D., ... van Duijn, C. M. (2011). Genome-wide association study identifies six new loci influencing pulse pressure and mean arterial pressure. Nature Genetics, 43, 1005-1011.

Wu, D., Molofsky, A. B., Liang, H. E., Ricardo-Gonzalez, R., Jouihan, H. A., Bando, J. K., ... Locksley, R. M. (2011). Eosinophils sustain adipose alternatively activated macrophages associated with glucose homeostasis. Science, 332, 243-247.

Yang, J., Loos, R. J., Powell, J. E., Medland, S. E., Speliotes, E. K., Chasman, D. I., ... Visscher, P. M. (2012). FTO genotype is associated with phenotypic variability of body mass index. Nature, 490, 267-272.

Ye, H., Hong, Q., Li, Y., Xu, X., Huang, Y. I., Xu, L., ... Duan, S. (2015). A lack of association between the IKZF2 rs12619285 polymorphism and coronary heart disease. Experimental and Therapeutic Medicine, 9, 1309-1313. 